\title{
The Association Between Chronic Immune-Mediated Inflammatory Diseases and Cardiovascular Risk
}

Jose Miguel Baena-Díez ${ }^{1,2,3}$, Maria Garcia-Gi1 ${ }^{4,5,6}$, Marc Comas-Cufi ${ }^{4,5}$, Rafel

Ramos $^{4,5,6,7}$, Daniel Prieto-Alhambra ${ }^{8,9}$, Betlem Salvador-González ${ }^{1,10}$, Roberto Elosua ${ }^{1}$, Irene R. Dégano ${ }^{1}$, Judith Peñafiel ${ }^{1}$, Maria Grau ${ }^{1,11 *}$

${ }^{1}$ REGICOR Study Group - Cardiovascular Epidemiology and Genetics, IMIM (Hospital del Mar Medical Research Institute), Barcelona, Spain

${ }^{2}$ La Marina Primary Care Centre and Primary Care Research Institute Jordi Gol, Catalan Institute of Health, Barcelona, Spain

${ }^{3}$ Consortium for Biomedical Research in Epidemiology and Public Health

(CIBERESP), Spain

${ }^{4}$ Institut Universitari d'Investigació en Atenció Primària Jordi Gol (IDIAP Jordi Gol), Spain

${ }^{5}$ ISV Research Group, Research Unit in Primary Care, Primary Care Services, Girona, Catalan Institute of Health (ICS), Spain

${ }^{6}$ TransLab Research Group, Department of Medical Sciences, School of Medicine, University of Girona, Girona, Spain

${ }^{7}$ Biomedical Research Institute, Girona (IdIBGi), ICS, Spain

${ }^{8}$ Musculoskeletal diseases Research Group (GREMPAL), Primary Care Research Institute Jordi Gol, Universitat Autònoma de Barcelona, Barcelona, Spain

${ }^{9}$ Musculoskeletal Pharmaco- and Device Epidemiology, Nuffield Department of

Orthopaedics, Rheumatology, and Musculoskeletal Sciences, University of Oxford, Oxford, United Kingdom

${ }^{10}$ Florida Sud Primary Care Centre and Primary Care Research Institute Jordi Gol, Catalan Institute of Health, L'Hospitalet de Llobregat, Spain

${ }^{11}$ University of Barcelona, Spain

*Corresponding author: Tel. +34 9331608 00; Fax. +34 9331607 96; email: mgrau@imim.es 


\begin{abstract}
Objective To examine the association between chronic immune-mediated diseases (rheumatoid arthritis, systemic lupus erythematosus, or the following chronic immunemediated inflammatory diagnoses groups: inflammatory bowel diseases, inflammatory polyarthropathies, systemic connective tissue disorders and spondylopathies) and the 6year coronary artery disease, stroke, cardiovascular disease incidence and overall mortality; and to estimate the population attributable fractions for all four end-points for each chronic immune-mediated inflammatory disease.

Methods Cohort study of individuals aged 35-85 years, with no history of cardiovascular disease from Catalonia (Spain). The coded diagnoses of chronic immune-mediated diseases and cardiovascular diseases were ascertained and registered using validated codes, and date of death was obtained from administrative data. Cox regression models for each outcome according to exposure were fitted to estimate hazard ratios (HR) in two models: (1) after adjustment for sex, age, cardiovascular risk factors and (2) further adjusted for drug use. Population attributable fractions were estimated for each exposure.
\end{abstract}

Results Data were collected from 991,546 participants. The risk of cardiovascular disease was increased in systemic connective tissue disorders [Model 1: HR=1.38 $(95 \%$ confidence interval=1.21-1.57) and $2: \mathrm{HR}=1.31(1.15-1.49)]$, rheumatoid arthritis $[\mathrm{HR}=1.43(1.26-1.62)$ and $\mathrm{HR}=1.31(1.15-1.49)]$ and inflammatory bowel diseases $[\mathrm{HR}=1.18(1.06-1.32)$ and $\mathrm{HR}=1.12(1.01-1.25)]$. The effect of anti-inflammatory treatment was significant in all instances $[\mathrm{HR}=1.50(1.24-1.81) ; \mathrm{HR}=1.47(1.23-1.75)$; $\mathrm{HR}=1.43$ (1.19-1.73), respectively]. The population attributable fractions for all three disorders were $13.4 \%, 15.7 \%$, and $10.7 \%$, respectively. 
Conclusion Systemic connective tissue disorders and rheumatoid arthritis conferred the highest cardiovascular risk and population impact, followed by inflammatory bowel diseases.

Keywords Cardiovascular disease, Inflammation, Arthritis, Connective Tissue

Diseases, Spondylarthritis, Inflammatory Bowel Diseases 


\section{Key messages}

\section{What is already known on this subject?}

Individuals diagnosed chronic immune-mediated inflammatory diseases present with increased CVD risk.

There are no comparable indicators to ascertain the cardiovascular risk associated with each chronic immune-mediated inflammatory disease due to the different inclusion criteria used in previous publications.

\section{What might this study add?}

Cardiovascular events and overall mortality risk was particularly pronounced in individuals diagnosed with systemic connective tissue disorders $[\mathrm{HR}=1.31(95 \%$ confidence interval=1.15-1.49) and $\mathrm{HR}=1.30(1.17-1.44)$, respectively] and rheumatoid arthritis $[\mathrm{HR}=1.31(1.15-1.49)$ and $\mathrm{HR}=1.31(1.18-1.46)$, respectively]. This risk was not explained by the higher prevalence of cardiovascular risk factors, nor by the use of DMARDs and anti-inflammatory drugs.

\section{How might this impact on clinical practice?}

The development of new tools for the prediction of cardiovascular events, which could incorporate CIID activity biomarkers, may help to reduce the incidence of such events. 


\section{INTRODUCTION}

The primary prevention of cardiovascular diseases (CVD) is a paramount priority of the public health agenda because it is the main cause of death in the developed world and is increasing alarmingly in developing countries. ${ }^{1}$ The common basis of CVD is atherosclerosis, an inflammatory degenerative process present throughout a person's lifetime. ${ }^{2}$ The pathogenesis of CVD in patients with chronic immune-mediated inflammatory diseases (CIID) is multi-factorial and is thought to result from an interaction of inflammation, metabolic factors, therapy, and disease-related factors. ${ }^{3}$

Individuals diagnosed with inflammatory bowel diseases, ${ }^{4}$ rheumatoid arthritis, ${ }^{5}$ systemic lupus erythematosus, ${ }^{6}$ systemic sclerosis, ${ }^{7}$ or ankylosing spondylitis ${ }^{8}$ have shown an increased CVD risk. However, straightforward comparison of the studies is difficult owing to the different inclusion criteria used. Better understanding of the association between these conditions and cardiovascular morbidities can help in early assessment and management of risk factors and may help to improve long-term outcomes in patients with CIID.

The objectives of the study were: (1) to ascertain whether 6-year risk of coronary artery disease, stroke, CVD incidence and overall mortality are associated with the diagnosis of rheumatoid arthritis, systemic lupus erythematosus, or the following CIID diagnoses groups: inflammatory bowel diseases, inflammatory polyarthropathies, systemic connective tissue disorders, and spondylopathies; and (2) to estimate the population-based burden of coronary artery disease, stroke, CVD, and mortality attributable to each of the studied CIID. 


\section{METHODS}

\section{Data sources}

The System for the Development of Research in Primary Care (SIDIAP) is an electronic medical records database derived from general practices of the Catalan Institute of Health in Catalonia (Spain). Data on approximately 5.8 million patients are recorded by general practitioners as part of patients' clinical records. Since approximately $80 \%$ of the population is registered with a general practitioner of the Catalan Institute of Health, SIDIAP is considered representative of the population in Catalonia. ${ }^{9}$ SIDIAP includes demographics, consultations with general practitioners, diagnoses, clinical variables, prescriptions, referrals to specialists, laboratory test results, and medications obtained from pharmacists. The International Classification of Diseases $10^{\text {th }}$ Edition (ICD-10) is used to code diagnoses and the Anatomical Therapeutic Chemical (ATC) Classification System is used for the classification of drugs. SIDIAP is also linked with hospital admissions data.

SIDIAP $^{\mathrm{Q}}$ is the subsample of individuals assigned to the $40 \%$ of general practitioners periodically selected to provide researchers with the best quality registry data. The methodology for selecting this subsample, which is representative of the Catalan population in terms of sex and age distribution, has been explained in detail elsewhere. ${ }^{9}$ The SIDIAP database has been validated for the study of the epidemiology of CVD by comparing the observed prevalence of cardiovascular risk factors and their association with the incidence of CVD with those of an epidemiological populationbased study with a standardized methodology. The present study was approved by the local ethics committees. 


\section{Study design}

We conducted a cohort study of individuals included in SIDIAP ${ }^{\mathrm{Q}}$. The inclusion period spanned 1 January 2007 through 31 December 2012. Participants were 35 to 85 years old and those who had a record documenting a past history of myocardial infarction, angina, stroke, transient ischemic attack, intermittent claudication or coronary revascularization procedures prior to the inclusion were excluded. Patients were followed up until they experienced the outcomes of interest, died, left the SIDIAP ${ }^{\mathrm{Q}}$ database (e.g. change of address) or the follow-up ended (31 December 2012), whichever came first.

\section{Definition of chronic immune-mediated inflammatory disease}

A pre-specified list of ICD-10 codes, set a priori and based on previous work in the field, ${ }^{10}{ }^{11}$ was used to screen SIDIAP $^{\mathrm{Q}}$ records in order to identify all patients with CIID and classify them in four groups: (1) inflammatory bowel disease, (2) inflammatory polyarthropathies, (3) systemic connective tissue disorders, and (4) spondylopathies (see the disorders included in each group in Supplementary Table 1). In addition, a complementary analysis was performed for the most prevalent single diagnoses: (5) rheumatoid arthritis and (6) systemic lupus erythematosus. We included individuals without a registered diagnosis who required a continued prescription for a medication that would normally be used to treat inflammatory bowel disease, rheumatoid arthritis or systemic lupus erythematosus, based on evidence-based recommendations. ${ }^{12-15}$ If an individual presented with more than two diagnoses, we considered the oldest one for the purpose of this analysis. Individuals who developed CIID during follow-up were considered non-exposed. Since the CIID considered in this study have physical features, 
adverse prognosis and the database has been previously validated ${ }^{10} 11$ diagnoses are expected to be accurately recorded.

\section{Identification of outcomes}

The end-points considered were: (1) coronary artery disease: incident myocardial infarction or angina; (2) incident stroke; (3) cardiovascular disease: coronary artery disease or stroke; and (4) all-cause mortality (Supplementary Table 2). All end-points were identified from the SIDIAP and Hospital Discharge registries. The methodology for case-finding used in the SIDIAP database has shown high validity and good representativeness of the population for use in epidemiological studies of CVD [9].

\section{Assessment of covariables}

Data were obtained from the database on sex, age, systolic and diastolic blood pressure, glucose, triglycerides, total, high density lipoprotein (HDL) and low-density lipoprotein (LDL) cholesterol, glycaemia, weight, and height at baseline.

Presence of cardiovascular risk factors was extracted from electronic records based on the presence of relevant codes (which follow the recommendations of the clinical practice guidelines of the Catalan Institute of Health) with a date prior to the inclusion of each participant: (1) Diabetes mellitus: ICD-10 categories E11, E12, E14, and subcategories thereof; (2) Hypertension: ICD-10 categories I10, I15, and subcategories thereof; (3) Hypercholesterolemia: ICD-10 category E78 and its subcategories, except for E78.3 and E78.6; (4) Smoking: ICD-10 category F17 for smokers or Z72.0 for exsmokers and (5) Obesity: ICD-10 category E66 and subcategories except for E66.1 and E66.2 [9]. Coronary artery disease risk was estimated in participants aged 35 to 74 
years with the Framingham-REGICOR function (Girona Heart Registry [Registre Gironí del Cor]) validated for the Spanish population. ${ }^{16}$ Participants were categorized into three treatment groups: disease-modifying anti-rheumatic drugs including biologic therapies (DMARD), anti-inflammatory drugs (i.e. non-steroidal anti-inflammatory drugs, glucocorticoids, other non-inflammatory drugs), and non-users of DMARD and anti-inflammatory drugs. Lipid-lowering and antihypertensive treatments were also considered. The drugs included in each group are summarized in Supplementary Table 3,4 and 5 .

\section{Statistical analysis}

We used 10 multiple imputations by chained equations ${ }^{17}$ to replace missing baseline values for total, HDL and LDL cholesterol, triglycerides, glycaemia, systolic and diastolic blood pressure, height, weight and MEDEA index (a deprivation index designed to capture sex-related inequalities). In addition to incorporating the missing-atrandom assumption, we compared case-complete results alone with the multiple imputations as a sensitivity analysis.

Continuous variables were summarized as mean (standard deviation) or median [interquartile range] when their distribution departed from normal, and categorical variables as proportions. The incidence of coronary artery disease, stroke and CVD events and overall mortality was estimated. We plotted the Kaplan-Meier curves for all four end-points by CIID diagnoses and in population with no CIID and we performed log-rank tests to estimate the differences between each diagnosis and CIID-free individuals. Bonferroni correction for multiple comparisons was applied, considering as significant a p-value $<0.002$. 
To assess the association between the CIID diagnoses and the 6-year incidence of coronary artery disease, stroke and CVD events or death, we adjusted two Cox regression models for each outcome. The first was adjusted for sex, age, systolic and diastolic blood pressure, total and HDL cholesterol, diabetes and smoking. The second was further adjusted for lipid-lowering, antihypertensive and rheumatic-specific drug use divided in three categories. The proportionality assumption was tested for all models (Supplementary Table 6). To test the robustness of case-definition, we performed several sensitivity analyses. In the first, we excluded patients with no record of a diagnosis but who required continued prescription of a medication normally used to treat inflammatory bowel disease, rheumatoid arthritis, or systemic lupus erythematosus, based on evidence-based recommendations. The second sensitivity analysis excluded those individuals who were receiving anti-inflammatory treatment (NSAIDs or DMARDs) before the CIID diagnosis. The third excluded those individuals with chronic diseases that may be related with both inflammation and cardiovascular risk (e.g., chronic kidney disease, sleep disorders, and liver disease). Also, as a sensitivity analysis, model 2 was adjusted using the Fine and Gray method for coronary artery disease, stroke and CVD outcomes and considering death as a competing event.

Finally, to estimate the burden of CVD and mortality attributable to each CIID, the PAF were estimated for all four end-points, using Levin's formula. We used the adjusted hazard ratios (HR) according to model 2 and the prevalence of every CIID diagnosis observed in our cohort. 


\section{RESULTS}

We included 991,546 individuals in our analysis, 467,494 (47.1\%) men, mean age 53 years (standard deviation=13). Inflammatory polyarthropathies was the most prevalent CIID diagnosis $(2.3 \%)$, followed by inflammatory bowel diseases $(1.0 \%)$, systemic connective tissue disorders $(0.5 \%)$ and spondylopathies $(0.2 \%)$ (Figure 1$)$. Additionally, $5552(0.6 \%)$ presented with rheumatoid arthritis and $664(0.07 \%)$ with systemic lupus erythematosus. Individuals with CIID were older, and more often presented with diabetes, hypertension, dyslipidemia, obesity and higher 10-year cardiovascular risk than those without CIID. Finally, the individuals with CIID were more frequently on treatment with statins, aspirin and DMARDs (Table 1).

Annual incidence rates for all end-points showed significantly higher rates in those diagnosed with CIID (Table 2). Additionally, the Kaplan-Meier curves showed significantly lower disease-free time (i.e., coronary artery disease, cerebrovascular diseases and CVD) and survival for all CIID diagnoses compared with CIID-free population, except for systemic lupus erythematosus and spondylopathies. Indeed, the latter only presented with higher risk for death (Figure 2).

The multivariable models showed that systemic connective tissue disorders and rheumatoid arthritis increased the risk of all end-points considered, particularly coronary artery disease for the first $[\mathrm{HR}=1.37$; (95\% confidence interval: $1.15-1.65)]$ and stroke for the latter $[\mathrm{HR}=1.33(1.11-1.60)]$. Among individuals with systemic lupus erythematosus, only the risk of mortality was significantly higher $[\mathrm{HR}=1.50 ;(1.03$ 2.18)], even though the group in which this disease is included (i.e. systemic connective tissue disorders) conferred an increased risk for all end-points. On the other hand, the group of inflammatory polyarthropathies only showed a marginally significant 
association with stroke $[\mathrm{HR}=1.09 ;(0.99-1.19)]$. Finally, inflammatory bowel diseases had a significant association with stroke and with overall mortality $[\mathrm{HR}=1.23$; (1.061.43) and $\mathrm{HR}=1.64 ;(1.51-1.76)$, respectively] (Figure 3). No relevant differences were found between the main analysis and any sensitivity analysis performed (Supplementary Tables 7-12).

The CIIDs with significant population impact on coronary artery disease, stroke and CVD were systemic connective tissue disorders (15.6\%, 9.1\%, and $13.4 \%$, respectively) and rheumatoid arthritis $(14.4 \%, 16.5 \%$, and $15.7 \%$, respectively). In addition, inflammatory bowel diseases presented significant PAF for stroke (18.7\%) and for cardiovascular diseases (10.7\%). Finally, the population impact on overall mortality was significant for all CIIDs, except for the spondylopathies, although the magnitude ranged from $39.0 \%$ for inflammatory bowel diseases to $3.4 \%$ for systemic lupus erythematosus (Figure 4).

\section{DISCUSSION}

Diagnoses of CIID confer an increased risk of cardiovascular disease and overall mortality. This risk was particularly pronounced in individuals diagnosed with systemic connective tissue disorders and rheumatoid arthritis. Overall mortality risk was increased in both groups of diseases. This greater risk was not explained by aging, the higher prevalence of cardiovascular risk factors observed in CIID individuals, nor by the use of DMARDs and anti-inflammatory drugs, which may have a role in the modulation of cardiovascular risk. Indeed, the differences in the estimated cardiovascular risk did not reflect higher incidence in individuals with CIID compared to those without, pointing out the effect of other variables not included in such 
estimates. Additionally, inflammatory bowel diseases raised the risk of stroke and the overall mortality risk.

\section{Risk of cardiovascular disease in chronic immune-mediated inflammatory disease}

Individuals with CIID were older and had a worse cardiovascular risk factor profile; however, the multivariable analysis adjusted for the relevant variables showed an added risk not explained by these cardiovascular risk-related variables. A previous study, which also analyzed data from electronic medical records, showed similar coronary artery disease and stroke incidence in individuals with systemic connective disorders, inflammatory polyarthropathies, and inflammatory bowel diseases. ${ }^{18}$ Additionally, we performed the same analysis separately for rheumatoid arthritis and for systemic lupus erythematosus, the most prevalent diseases within both groups, and therefore the most reported in the literature. As previously described, rheumatoid arthritis showed a significant risk in all four end-points, even after adjustment for drug therapy. ${ }^{19}$ Lazzerini et al. pointed out that a higher risk of rhythm disturbances, particularly tachiarrythmias, in such individuals may significantly contribute to the high cardiovascular morbidity and mortality. ${ }^{20}$ Systemic lupus erythematosus only showed a significant association with overall mortality. However, the systematic review performed by Schoenfeld et al. showed that systemic lupus erythematosus at least doubled cardiovascular risk. ${ }^{21}$ Concurring with Andersohn et al., individuals with inflammatory bowel diseases presented higher risk of stroke. ${ }^{22}$ On the other hand, the association between these diseases and coronary artery disease has not been totally clarified. Similar to previous reports, we did not find an increased risk of coronary artery disease in these individuals; ${ }^{18}$ however, this association was significant in the 
analysis of a nation-wide, population-based Danish cohort. ${ }^{23}$ Finally, individuals with spondylopathies did not present with significantly higher risk for any of the end-points analyzed.

\section{Prevention of cardiovascular disease in chronic immune-mediated inflammatory}

\section{disease}

The most-used treatments for controlling CIID, and particularly rheumatoid arthritis, the most thoroughly described, may have antagonistic effects on cardiovascular risk. The DMARDs drugs may confer a cardioprotective effect mostly attributed to the inhibition of systemic inflammation. ${ }^{24}$ However, Aviña-Zubieta et al. described a dual effect of glucocorticoids on myocardial infarction risk in individuals with rheumatoid arthritis: an immediate effect mediated through current dosage and a long-term effect of cumulative exposure. ${ }^{25}$ In addition, del Rincón et al. pointed out that daily doses higher than $8 \mathrm{mg}$ were associated with higher cardiovascular and overall mortality in such individuals. ${ }^{26}$ Recently, O’Neill et al. showed in a randomized clinical trial the beneficial effect of certain anti-inflammatory treatments that modulate the tumour necrosis factor- $\alpha$ pathway (e.g. methortrexate + infliximab), to restore the beneficial effects of HDL on the vasculature. ${ }^{27}$

To minimize the confounder effect of such variables, Model 2 of the present analysis was adjusted for drug use, divided in three categories (DMARDs, anti-inflammatory drugs, and non-DMARD non-anti-inflammatory drugs). Indeed, further pharmacoepidemiology studies are needed to ascertain the effect of antirheumatic treatments in all CIID diagnoses. 
A reduction in the number of individuals diagnosed with systemic connective tissue disorders and rheumatoid arthritis may have a moderate population impact on preventing coronary artery disease, stroke and CVD. However, the diagnosis of such CIIDs together with an adverse cardiovascular profile may have an additive effect on the risk of coronary artery disease and CVD. The most recent European League Against Rheumatism (EULAR) guideline promotes a proactive management of cardiovascular risk in individuals with inflammatory polyarthritis and spondylopathies. The objective is to control not only systemic inflammation, but also cardiovascular risk factors. ${ }^{28}$ These recommendations have also been proposed for individuals with systemic lupus erythematosus ${ }^{29}$ but could probably be extended to individuals with any systemic connective tissue disorder. In addition, the development of new tools for the prediction of cardiovascular events, which could incorporate CIID activity biomarkers, may help to reduce the incidence of such events. ${ }^{24} 30$

\section{Characteristics and limitations of the study}

Our study has several limitations. To avoid misclassification bias, we used medical diagnosis of CIID extracted from SIDIAP, a primary care database. Although this source of data may contain underreporting, the validity of SIDIAP coding for ICD-10 diagnosis of cardiovascular and rheumatic diseases has been documented. ${ }^{10}{ }^{11}$ Indeed, the results of the sensitivity analyses confirm the robustness of the case-definition. In addition, the representativeness of data ensures the generalizability of the results. The covariates of the present analysis had missing values (ranging from $9 \%$ to $78 \%$ ) that could influence the results. To avoid selection bias, wherever the population with missing data differed from those with complete data, we imputed the missing values for 
continuous variables instead of excluding records with missing data. The appropriateness of performing multiple imputations depends not only on the percentage and mechanism of missing values but also on the number of complete observations used in the imputation process. In our study, 218,140 complete cases were available to impute variables with missing values. Exposure variables (i.e. CIID diagnoses) and outcomes did not present missing values. In our study, we analyzed the two most frequent single CIID diagnoses (rheumatoid arthritis and systemic lupus erythematosus) in the population and in the literature. In addition, we provided broad definitions of CIID diagnosis (i.e., inflammatory bowel diseases, inflammatory polyarthropathies, systemic connective tissue disorders and spondylopathies). This approach, proposed by Dregan et al., allowed for comparison of results. ${ }^{18}$ Finally, the present analysis did not consider variables such as severity or elapsed time between CIID diagnosis and the incidence of cardiovascular events or death. Indeed, the use of broad CIID definitions may complicate the use of such variables due to the number and the heterogeneity of diseases included in every group.

The results of our study confirm the high cardiovascular risk conferred by previous studies exploring CIID incidence with lower sample sizes. ${ }^{4-8} 22$ However, some biases inherent to the observational design of the study should be considered. On the one hand, additional confounding factors not controlled in the present analysis are likely to play a key role in cardiovascular risk of individuals with CIID. On the other hand, the effect of cardiovascular and rheumatic disease-specific treatments merits the development of pharmacoepidemiology studies considering each CIID's specific variables to ascertain the best way to prevent cardiovascular diseases in such individuals. 


\section{CONCLUSION}

Systemic connective tissue disorders and rheumatoid arthritis were the CIID diagnoses with the highest 6-year risk of coronary artery disease or any cardiovascular event and the greatest population impact. Diagnosis of inflammatory bowel diseases (i.e. ulcerative colitis and Crohn disease) conferred a significant risk and PAF for stroke. Regarding overall mortality, all CIID diagnoses except spondylopathies increased 6year mortality.

Conflict of interest statement The authors report no conflict of interest Acknowledgments The authors are grateful to the System for the Development of Research in Primary Care (SIDIAP) database and to Dr. Albert Escola Campabadal for the expert review on chronic immune mediated inflammatory disorders, and also appreciate the revision of the English text by Elaine Lilly $\mathrm{PhD}$.

Sources of funding This work has been supported by grants from the Instituto de Salud Carlos III FEDER (CP12/03287; HERACLES RD12/0042; RedIAPP RD12/0005), AGAUR (2014 SGR 240). IRD was funded by the RECERCAIXA Program, Obra Social "La Caixa" [RE087465].

Author's contributions JMBD, MGG, MCC, RR, and MG conceived and designed the study. JMBD, MGG, MCC, RR, DPA, RE and MG acquired, analyzed, or interpreted the data. JMBD, BS, IRD and MG drafted the manuscript. MGG, MCC and JP carried out the statistical analysis. JMBD and MG supervised the study. MG had full 
access to all of the data in the study and takes responsibility for the integrity of the data and the accuracy of the data analysis.

Ethics approval Parc de Salut Mar Ethics Committee.

\section{REFERENCES}

1 World Health Statistics [Homepage on the Internet]. Geneva: World Health Organization; 2013 [Cited November 27, 2015]. Available from: https://apps.who.int/infobase/

2 Hansson GK, Libby P, Tabas I. Inflammation and plaque vulnerability. J Intern Med 2015;278:483-93.

3 Mason JC, Libby P. Cardiovascular disease in patients with chronic inflammation: mechanisms underlying premature cardiovascular events in rheumatologic conditions. Eur Heart J 2015;36:482-9c.

4 Yarur AJ, Deshpande AR, Pechman DM, et al. Inflammatory bowel disease is associated with an increased incidence of cardiovascular events. $A m J$ Gastroenterol 2011;106:741-7.

5 Avina-Zubieta JA, Thomas J, Sadatsafavi M, et al. Risk of incident cardiovascular events in patients with rheumatoid arthritis: a meta-analysis of observational studies. Ann Rheum Dis 2012;71:1524-9.

6 Westerweel PE, Luyten RK, Koomans HA, et al. Premature atherosclerotic cardiovascular disease in systemic lupus erythematosus. Arthritis Rheum 2007;56:1384-96.

7 Man A, Zhu Y, Zhang Y, et al. The risk of cardiovascular disease in systemic sclerosis: a population-based cohort study. Ann Rheum Dis 2013;72:1188-93. 
8 Papagoras C, Voulgari PV, Drosos AA. Atherosclerosis and cardiovascular disease in the spondyloarthritides, particularly ankylosing spondylitis and psoriatic arthritis. Clin Exp Rheumatol 2013;31:612-20.

9 Ramos R, Balló E, Marrugat J, et al. Validity for use in research on vascular diseases of the SIDIAP (Information System for the Development of Research in Primary Care): the EMMA study. Rev Esp Cardiol (Engl Ed) 2012;65:29-37.

10 Fina-Aviles F, Medina-Peralta M, Mendez-Boo L, et al. The descriptive epidemiology of rheumatoid arthritis in Catalonia: a retrospective study using routinely collected data. Clin Rheumatol 2016;35:751-7.

11 Muñoz-Ortego J, Vestergaard P, Rubio JB, et al. Ankylosing spondylitis is associated with an increased risk of vertebral and nonvertebral clinical fractures: a population-based cohort study. J Bone Miner Res 2014;29:1770-6.

12 Smolen JS, Landewé R, Breedveld FC, et al. EULAR recommendations for the management of rheumatoid arthritis with synthetic and biological diseasemodifying antirheumatic drugs: 2013 update. Ann Rheum Dis 2014;73:492-509.

13 Lichtenstein GR, Hanauer SB, Sandborn WJ; Practice Parameters Committee of American College of Gastroenterology. Management of Crohn's disease in adults. Am J Gastroenterol 2009;104:465-83.

14 Kornbluth A, Sachar DB; Practice Parameters Committee of the American College of Gastroenterology. Ulcerative colitis practice guidelines in adults: American College Of Gastroenterology, Practice Parameters Committee. Am J Gastroenterol 2010;105:501-23.

15 Bertsias G, Ioannidis JP, Boletis J, et al. EULAR recommendations for the management of systemic lupus erythematosus. Report of a Task Force of the 
EULAR Standing Committee for International Clinical Studies Including Therapeutics. Ann Rheum Dis 2008;67:195-205.

16 Marrugat J, Subirana I, Comín E, et al. Validity of an adaptation of the Framingham cardiovascular risk function: the VERIFICA Study. J Epidemiol Community Health 2007;61:40-47.

17 White IR, Royston P, Wood AM. Multiple imputation using chained equations: Issues and guidance for practice. Stat Med 2011;30:377-99.

18 Dregan A, Charlton J, Chowienczyk P, et al. Chronic inflammatory disorders and risk of type 2 diabetes mellitus, coronary heart disease, and stroke: a populationbased cohort study. Circulation 2014;130:837-44.

19 Crowson CS, Liao KP, Davis JM 3rd, et al. Rheumatoid arthritis and cardiovascular disease. Am Heart J. 2013;166:622-628.

20 Lazzerini PE, Capechi PL, Laghi-Pasini F. Systemic inflammation and arrhythmic risk: lessons from rheumatoid arthritis. Eur Heart J 2016 (in press)

21 Schoenfeld SR, Kasturi S, Costenbader KH. The epidemiology of atherosclerotic cardiovascular disease among patients with SLE: a systematic review. Semin Arthritis Rheum 2013;43:77-95.

22 Andersohn F, Waring M, Garbe E. Risk of ischemic stroke in patients with Crohn's disease: a population-based nested case-control study. Inflamm Bowel Dis 2010;16:1387-92.

23 Rungoe C, Basit S, Ranthe MF, et al. Risk of ischaemic heart disease in patients with inflammatory bowel disease: a nationwide Danish cohort study. Gut 2013;62:689-94. 
24 Hollan I, Dessein PH, Ronda N, et al. Prevention of cardiovascular disease in rheumatoid arthritis. Autoimmun Rev 2015;14:952-69.

25 Aviña-Zubieta JA, Abrahamowicz M, De Vera MA, et al. Immediate and past cumulative effects of oral glucocorticoids on the risk of acute myocardial infarction in rheumatoid arthritis: a population-based study. Rheumatology (Oxford) 2013;52:68-75.

26 del Rincón I, Battafarano DF, Restrepo JF, et al. Glucocorticoid dose thresholds associated with all-cause and cardiovascular mortality in rheumatoid arthritis. Arthritis Rheumatol 2014;66:264-72.

27. O'Neill F, Charakida M, Topham E, et al. Anti-inflammatory treatment improves high-density lipoprotein function in rheumatoid arthritis. Heart. 2016 (in press)

28 Peters MJ, Symmons DP, McCarey D, et al. EULAR evidence-based recommendations for cardiovascular risk management in patients with rheumatoid arthritis and other forms of inflammatory arthritis. Ann Rheum Dis 2010;69:32531.

29 Mosca M, Tani C, Aringer M, et al. European League Against Rheumatism recommendations for monitoring patients with systemic lupus erythematosus in clinical practice and in observational studies. Ann Rheum Dis 2010;69:1269-74.

30 Solomon DH, Greenberg J, Curtis JR, et al. Derivation and internal validation of an expanded cardiovascular risk prediction score for rheumatoid arthritis: a Consortium of Rheumatology Researchers of North America Registry Study. Arthritis Rheumatol 2015;67:1995-2003. 


\section{Figure legends}

Figure 1 Participants flow-chart. CAD: Coronary artery disease. CIID: Chronic immune mediate inflammatory disease. CVD: Cardiovascular disease.

Figure 2 Survival curves for 6-year incidence of coronary artery disease (A), cerebrovascular disease (B), cardiovascular disease (C), and overall mortality (D) according to CIID diagnoses and in CIID-free population. Log-rank test p-values have been computed for each CIID diagnosis and CIID-free individuals.

Figure 3 Hazard ratios for 6-year incidence of coronary artery disease (A), cerebrovascular disease (B), cardiovascular disease (C), and overall mortality (D) among participants with CIID diagnosis compared with those without CIID. Model 1 has been adjusted by age, sex, smoking status, total cholesterol, HDL cholesterol, systolic blood pressure and diastolic blood pressure. Model 2 has been further adjusted for statins, hypertensive drugs and rheumatic disease-specific treatments divided in 3 categories: DMARDs users, other anti-inflammatory drugs, not exposed to any of them.

Figure 4 Population attributable fraction for 6-year incidence of coronary artery disease (A), cerebrovascular disease (B), cardiovascular disease (C), and overall mortality (D) for different chronic immune-mediated diseases. Bars are ordered according to the magnitude of the population-attributable fraction for each group of chronic immune-mediated disease diagnoses. 
Table 1 Baseline characteristics of participants according to diagnosis of chronic immune-mediated inflammatory diseases

\begin{tabular}{|c|c|c|c|c|c|}
\hline & $\begin{array}{l}\text { Inflammatory bowel } \\
\text { diseases }\end{array}$ & $\begin{array}{l}\text { Inflammatory } \\
\text { polyarthropathies }\end{array}$ & $\begin{array}{l}\text { Systemic connective } \\
\text { tissue disorders }\end{array}$ & Spondylopathies & CIID-free \\
\hline $\mathrm{N}(\%)$ & $9544(1.0)$ & $22779(2.3)$ & $4491(0.5)$ & $2243(0.2)$ & $952489(96.1)$ \\
\hline Age, mean (SD) & $56(14)$ & $60(12)$ & $63(14)$ & $55(12)$ & $52(13)$ \\
\hline Sex (men), n (\%) & $4162(44)$ & $14371(63)$ & $890(20)$ & $1185(53)$ & $446886(47)$ \\
\hline Diabetes, n (\%) & $807(10)$ & $2806(13)$ & $437(11)$ & $205(10)$ & $61131(7)$ \\
\hline Glycemia (mg/dl), median [IQR] & $92[82-104]$ & $95[85-108]$ & $91[82-102]$ & 92 [83-104] & $91[82-103]$ \\
\hline Hypertension, $\mathrm{n}(\%)$ & $2760(29)$ & $9633(42)$ & $1801(40)$ & $664(30)$ & $182170(19)$ \\
\hline Systolic blood pressure (mmHg), mean (SD) & $129(16)$ & $133(17)$ & $131(17)$ & $129(16)$ & $128(16)$ \\
\hline Diastolic blood pressure (mmHg), mean (SD) & $77(10)$ & $78(10)$ & $76(9)$ & $77(10)$ & $77(10)$ \\
\hline Dyslipidemia, n (\%) & $2645(28)$ & $8068(35)$ & $1493(33)$ & $581(26)$ & $174995(18)$ \\
\hline Total cholesterol (mg/dl), mean (SD) & $206(37)$ & $208(37)$ & $208(37)$ & $207(37)$ & $206(36)$ \\
\hline HDL cholesterol (mg/dl), mean (SD) & $57(15)$ & $55(15)$ & $61(15)$ & $56(15)$ & $57(15)$ \\
\hline LDL cholesterol (mg/dl), mean (SD) & $125(33)$ & $126(33)$ & $125(33)$ & $127(34)$ & $125(32)$ \\
\hline Triglycerides (mg/dl), median [IQR] & $103[74-143]$ & $113[81-161]$ & 99 [73-136] & $103[74-145]$ & 98 [71-138] \\
\hline Smoker, n (\%) & $3448(36)$ & $8763(38)$ & $998(22)$ & $921(41)$ & $294541(31)$ \\
\hline Obesity, n (\%) & $3226(34)$ & $9361(41)$ & $1608(36)$ & $763(34)$ & $278919(29)$ \\
\hline Body mass index, mean (SD) & $28.0(4.8)$ & $28.9(4.8)$ & $28.1(4.9)$ & $28.1(4.7)$ & $27.6(4.6)$ \\
\hline Statins, n (\%) & $1246(13)$ & $3870(17)$ & $903(20)$ & $296(13)$ & $85201(9)$ \\
\hline Aspirin, n (\%) & $425(4)$ & $1468(6)$ & $395(9)$ & $103(5)$ & $28281(3)$ \\
\hline Disease-modifying antirheumatic drugs, $\mathrm{n}(\%)$ & $737(8)$ & $1248(5)$ & $592(13)$ & $121(5)$ & $2013(0.2)$ \\
\hline Anti-hypertensive treatment, $\mathrm{n}(\%)$ & $2683(28)$ & $9006(40)$ & $1844(41)$ & $634(28)$ & $171004(18)$ \\
\hline 10-year cardiovascular risk (\%), median [IQR] & $2.4[1.3-4.4]$ & $3.6[2.0-6.6]$ & $2.3[1.4-4.0]$ & $2.7[1.5-4.9]$ & $2.1[1.1-3.7]$ \\
\hline
\end{tabular}

CIID, Chronic immune-mediated inflammatory disease; IQR, Interquartile range; SD, Standard deviation 


\begin{tabular}{|c|c|c|c|c|c|c|c|c|c|c|}
\hline & \multicolumn{2}{|c|}{$\begin{array}{l}\text { Inflammatory bowel } \\
\text { diseases } \\
\mathrm{N}=9544\end{array}$} & \multicolumn{2}{|c|}{$\begin{array}{l}\text { Inflammatory } \\
\text { polyarthropathies } \\
\mathrm{N}=22779\end{array}$} & \multicolumn{2}{|c|}{$\begin{array}{l}\text { Systemic connective } \\
\text { tissue disorders } \\
\mathrm{N}=4491\end{array}$} & \multicolumn{2}{|c|}{$\begin{array}{l}\text { Spondylopathies } \\
\mathrm{N}=2243\end{array}$} & \multicolumn{2}{|c|}{$\begin{array}{l}\text { CIID-free } \\
\mathrm{N}=952489\end{array}$} \\
\hline & $\mathrm{n}$ & $\begin{array}{l}\text { Annual incidence } \\
(95 \% \mathrm{CI})\end{array}$ & $\mathrm{n}$ & $\begin{array}{l}\text { Annual incidence } \\
(95 \% \mathrm{CI})\end{array}$ & $\mathrm{n}$ & $\begin{array}{l}\text { Annual incidence } \\
(95 \% \mathrm{CI})\end{array}$ & $\mathrm{n}$ & $\begin{array}{l}\text { Annual incidence } \\
(95 \% \mathrm{CI})\end{array}$ & $\mathrm{n}$ & $\begin{array}{l}\text { Annual incidence } \\
(95 \% \text { CI })\end{array}$ \\
\hline $\begin{array}{l}\text { Coronary artery } \\
\text { disease }\end{array}$ & 174 & $\begin{array}{l}3.23 \\
(2.75 \text { to } 3.71)\end{array}$ & 597 & $\begin{array}{l}4.61 \\
(4.24 \text { to } 4.98)\end{array}$ & 122 & $\begin{array}{l}4.84 \\
(3.98 \text { to } 5.70)\end{array}$ & 43 & $\begin{array}{l}3.34 \\
(2.34 \text { to } 4.34)\end{array}$ & 12841 & $\begin{array}{l}2.35 \\
(2.31 \text { to } 2.39)\end{array}$ \\
\hline Stroke & 177 & $\begin{array}{l}3.28 \\
(2.80 \text { to } 3.77)\end{array}$ & 519 & $\begin{array}{l}3.99 \\
(3.65 \text { to } 4.34)\end{array}$ & 118 & $\begin{array}{l}4.66 \\
(3.82 \text { to } 5.50)\end{array}$ & 29 & $\begin{array}{l}2.24 \\
(1.43 \text { to } 3.06)\end{array}$ & 10665 & $\begin{array}{l}1.95 \\
\text { (1.91 to } 1.99)\end{array}$ \\
\hline $\begin{array}{l}\text { Cardiovascular } \\
\text { disease }\end{array}$ & 344 & $\begin{array}{l}6.43 \\
(5.75 \text { to } 7.11)\end{array}$ & 1090 & $\begin{array}{l}8.49 \\
(7.99 \text { to } 9.00)\end{array}$ & 237 & $\begin{array}{l}9.49 \\
(8.28 \text { to } 10.70)\end{array}$ & 71 & $\begin{array}{l}5.54 \\
(4.25 \text { to } 6.83)\end{array}$ & 23009 & $\begin{array}{l}4.24 \\
(4.18 \text { to } 4.29)\end{array}$ \\
\hline Overall mortality & 650 & $\begin{array}{l}11.96 \\
(11.04 \text { to } 12.88)\end{array}$ & 1403 & $\begin{array}{l}10.69 \\
(10.13 \text { to } 11.25)\end{array}$ & 362 & $\begin{array}{l}14.16 \\
(12.70 \text { to } 15.62)\end{array}$ & 95 & $\begin{array}{l}7.31 \\
(5.84 \text { to } 8.78)\end{array}$ & 28723 & $\begin{array}{l}5.23 \\
(5.17 \text { to } 5.29)\end{array}$ \\
\hline
\end{tabular}

CIID, Chronic immune-mediated inflammatory disease 
Figure 1.

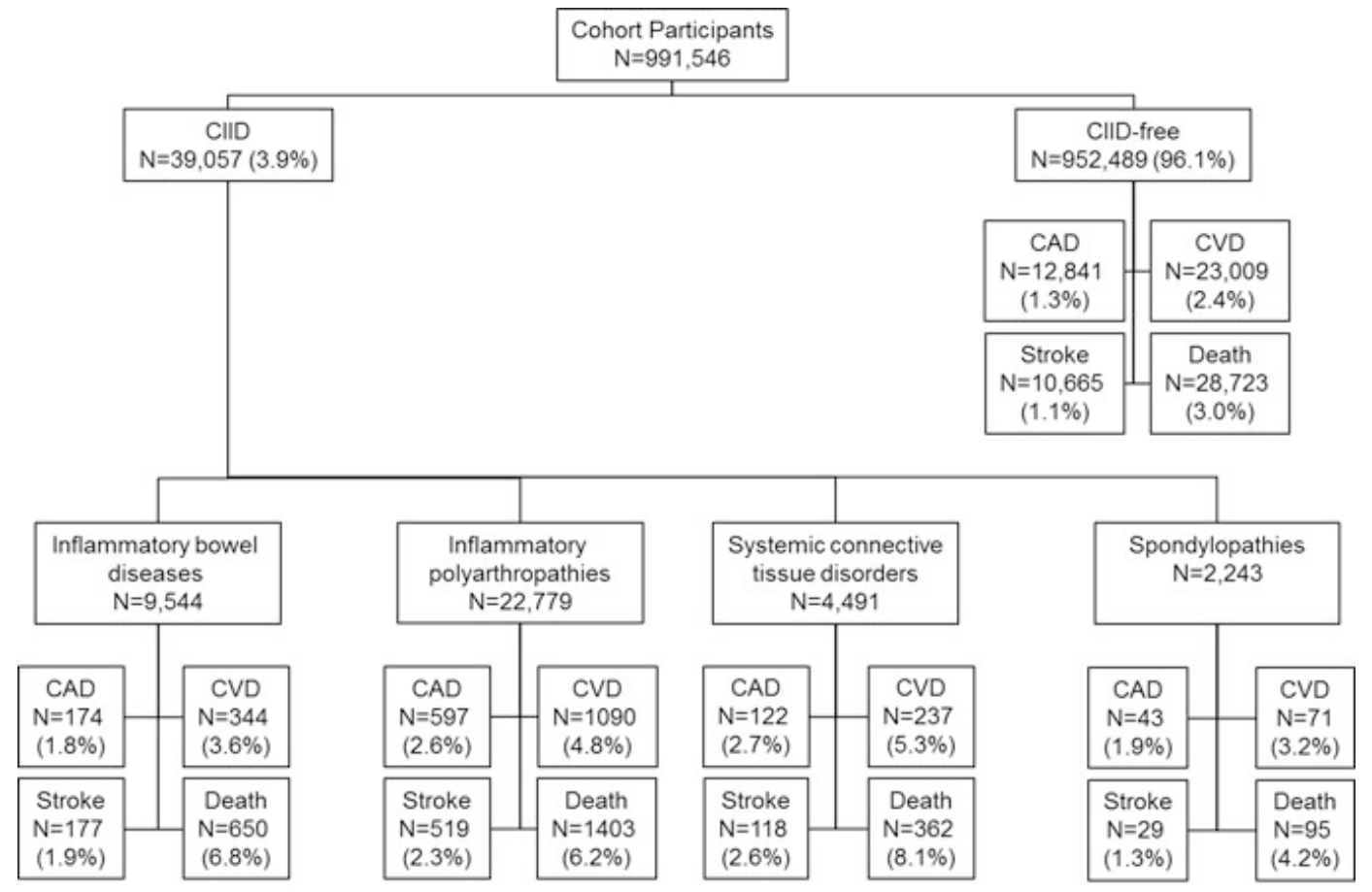




\section{Figure 2.}

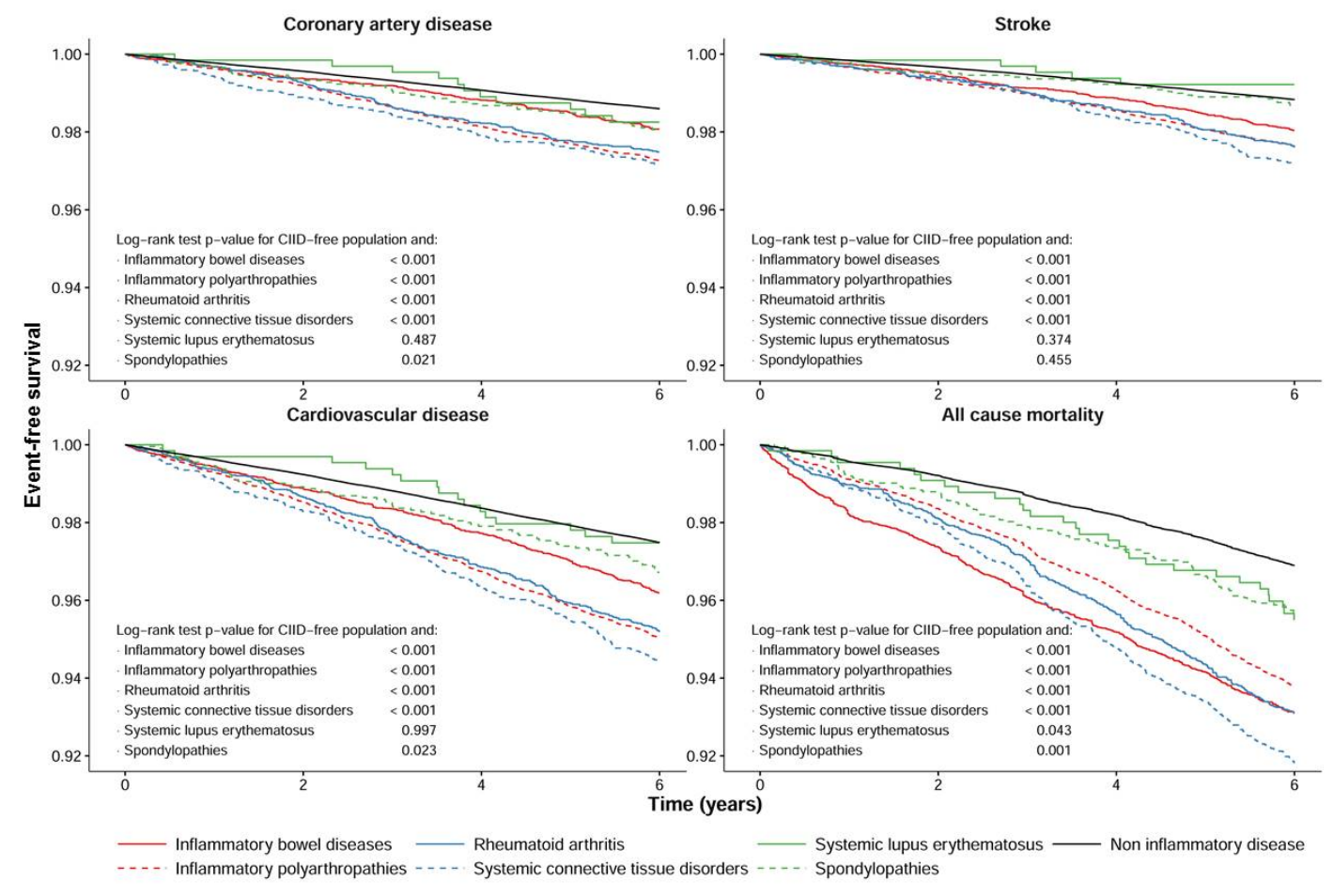




\section{Figure 3.}

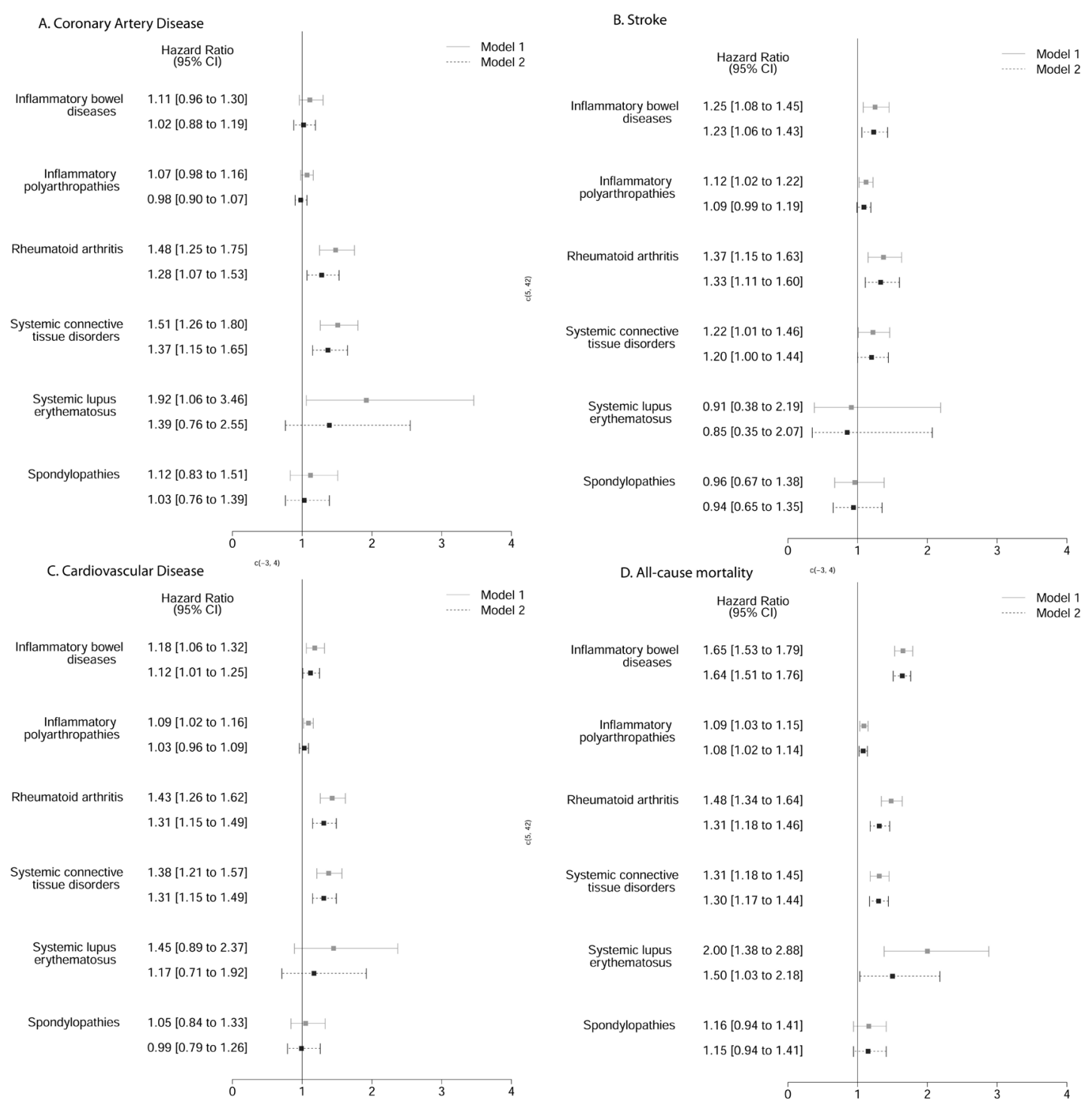




\section{Figure 4.}

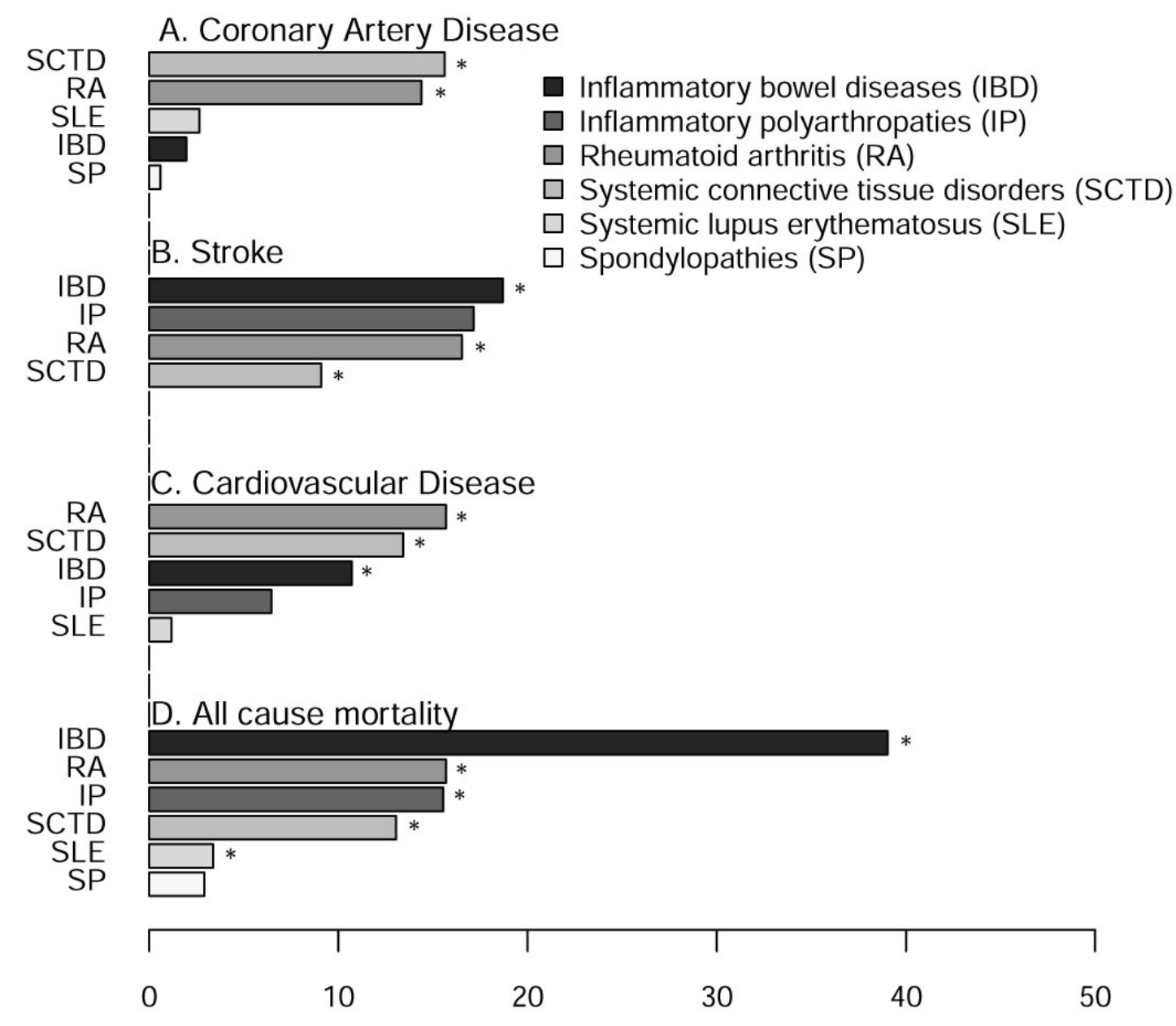

Population attributable fraction (\%) 